(C) 2017 IEEE. Personal use of this material is permitted. Permission from IEEE must be obtained for all other uses, in any current or future media, including reprinting/republishing this material for advertising or promotional purposes, creating new collective works, for resale or redistribution to servers or lists, or reuse of any copyrighted component of this work in other works. 


\title{
Sliding-Mode Observer Based Voltage-Sensorless Model Predictive Power Control of PWM Rectifier Under Unbalanced Grid Condition
}

\author{
Haitao Yang, Student Member, IEEE, Yongchang Zhang, Member, IEEE, Jiejunyi Liang, Jihao Gao, Paul D. \\ Walker and Nong Zhang
}

\begin{abstract}
A sliding-mode grid voltage observer (SMGVO) is proposed and experimentally verified in this paper for voltagesensorless operation under an unbalanced network. Fundamental positive sequence component (FPSC) and fundamental negative sequence component (FNSC) are inherently separated in the observer without employing any additional filters. Due to embedded filtering effect, high frequency chattering and harmonic ripples can be well suppressed. Additionally, DC component can be completely rejected. As a result, DC offset would not cause fundamental frequency oscillations in magnitude and frequency of the estimated FPSC and FNSC. Owing to the predictive ability of SMGVO, one-step delay can be directly compensated using state variables in the observer. By combining estimation and prediction into one stage, the designed SMGVO turns out to be a compact solution for finite control set-model predictive power control (FCS-MPPC) without voltage sensors. Theoretical proof is derived to verify that FPSC and FNSC can be accurately estimated and separated. Experimental results obtained from a two-level PWM rectifier confirm the effectiveness of the whole control system.
\end{abstract}

Index Terms-Predictive power control, unbalanced grid, voltage observer, voltage sensorless

\section{INTRODUCTION}

Generally, accurate power control of three-phase voltage source rectifier (VSR) requires information of grid voltage and current. Hence, sensors for measuring grid voltage and current are usually installed in practical application. However, sensors increases hardware cost and mounting space [1]. On the contrary, sensorless control has merits of better environment adaptability, lower cost, etc. Additionally, sensorless control schemes can be used as backup so that the system can continue operation during sensor-fault conditions. In the existing literature, both voltage sensorless control [2]-[5] and current sensorless control [6], [7] have been investigated for power converters. For grid-tied converters, grid current is usually regulated to satisfy control objectives. The requirement of high current control bandwidth would impose a high demand on designing a current estimator. Additionally, current sensors are usually required for overload protection [8]. On the other hand, grid voltages usually operate at steady-state and hence a good estimation can be easily obtained. During the past decade, many voltage sensorless methods have been proposed.

Among different sensorless control schemes, the concept of virtual flux (VF) is popular, which has been widely investigated for various control methods (e.g., direct power control (DPC), flux oriented control and predictive power control) [1],
[2], [9]. However, the pure integrator suffers issues of DC drift and initial bias in practical application. To address these problems, low pass filter (LPF) [2], [9] or second order generalized integrator (SOGI) [8], [10], etc. is typically adopted to emulate the function of integrator at fundamental frequency. However, incorporation of these filters in an open-loop estimator would inevitably cause a long delay during transient process, such as startup process and sudden voltage sag [1], [11]. In [1], a method of fast initial bias compensation is proposed based on time domain analysis assuming an ideal grid voltage. During normal operation, the DC drift is solved by calculating average value of VF within each fundamental cycle. As DC bias updating rate equals fundamental frequency of grid voltage, dynamic performance may be affected during sudden voltage change. To avoid relatively slow converging rate of first order LPF, a fast integration scheme is proposed in [11] aiming at faster dynamic responses during transient process. However, most of those schemes cannot directly provide separation of fundamental positive sequence component (FPSC) and fundamental negative sequence component (FNSC) under unbalanced grid condition. To solve this problem, a SOGI based VF estimator with inherent sequence separation is proposed in [10]. For better dynamic performance, four parallel SOGIs instead of cascaded structure are utilized for both converter voltage and current. The FPSC and FNSC are then extracted from filtered signal and its quadrature value. As SOGI based sequence separation presents nonzero gain at zero frequency, DC drift due to imperfect measurement, grid faults, etc. would inevitably cause fundamental frequency oscillation in magnitude and phase of estimated grid voltage [12].

Apart from VF, other schemes, such as state observer [3], [4] and Kalman filter (KF) [13], [14] have been also investigated for voltage sensorless control. Unlike open-loop structure of VF estimators, both state observer and KF are closed-loop estimators. The feedback of current tracking error could enhance estimator's stability and prevent saturation caused by DC drift. In [3], a notch filter is required after voltage estimation for proper operation under unbalanced grid conditions. While state observer presented in [4] can directly offer FPSC and FNSC without using a filter. Though stability analysis in [4] shows that the estimation error of grid voltage can converge to zero, the analytic relationship between estimated value and actual value is still unclear, complicating further theoretical analysis of observer's performance. For KF, satisfactory noise immunity is its major merit, but higher computational burden 
is usually referred as the main drawback. Additionally, prior knowledge of measurement and process noises are required for parameter tuning, which can not be easily derived [14].

Though sliding mode theory has been widely investigated in the design of controllers for PWM rectifiers [15], [16], relatively little research on sliding mode observers has been carried out for grid voltage estimation, especially under unbalanced grid conditions. The main reason may be the potential problem of high frequency chattering, which would introduce undesired ripple components in the control system. In [17], a sliding-mode grid voltage observer (SMGVO) is proposed to extract FPSC. As estimated grid voltage is reconstructed from switching function, LPFs are employed to filter out high frequency chattering and attenuate grid voltage harmonics. However, employment of two cascaded LPFs after the observer would deteriorate dynamic performance. Additionally, LPF can not cancel DC drift in estimation. As studied in [12], if phase-locked loop (PLL) is implemented, rejection of DC component is desirable to remove fundamental frequency oscillations in estimated phase, frequency and magnitude. In this paper, an improved SMGVO will be developed without these issues.

After getting the estimation of grid voltage, the next step is to implement a suitable power control scheme. Recently, finite control set-model predictive control (FCS-MPC) has been widely researched for high performance control of power converters under both ideal and unbalanced grid condition [18][23]. Compared with two well established control methods, i.e., voltage oriented control (VOC) and switching table based DPC [24], FCS-MPC presents superiority in handling multiobjective control and system constraints [25], [26]. In FCSMPC, no pulse width modulation is required and the optimal switching states can be directly selected by minimizing a predefined cost function [19], [23], [26]. Due to the lack of a modulator, only discrete voltage vectors are available in FCS-MPC, which consist of significant ripples. In real world, due to imperfect measurement and inaccurate system parameters, ripples of input voltage to the observer can not be perfectly compensated by the measured responses [27]. Hence, the voltage estimator must be designed with good capacity to reject these disturbances. So far, little research has been investigated for FCS-MPC operating under unbalanced grid conditions without voltage sensors.

In this paper, a SMGVO is designed for operation under unbalanced grid condition. Analytical results confirm stability and accuracy of the designed observer. Based on the estimation of FPSC and FNSC from SMGVO, FCS-model predictive power control (FCS-MPPC) is implemented that can properly operate under an unbalanced network without voltage sensors. The main contributions of this work include:

1) A sliding mode grid voltage observer is designed and analyzed, which has not been fully investigated in the existing literature for grid voltage estimation, especially under unbalanced grid condition. Due to embedded filtering effect and sophisticated design, problem of high frequency chattering is avoided.

2) Separation of FPSC and FNSC is inherently achieved within the observer. Hence, no cascaded filters, such as

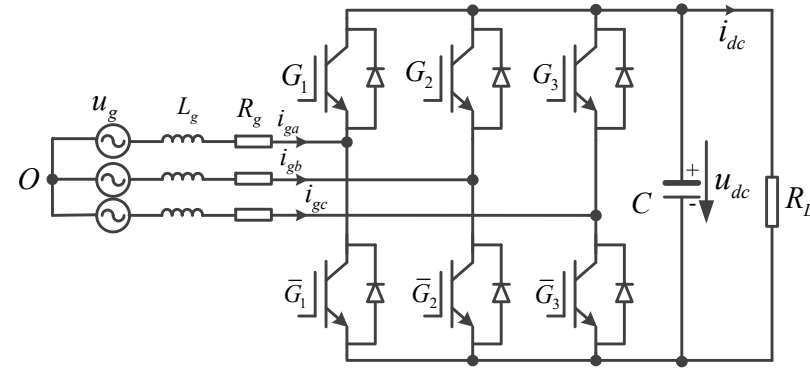

Fig. 1. Topology of a two-level VSR.

notch filters or SOGIs, are necessary in the algorithm.

3) DC offset is removed in the estimation, which can eliminate fundamental frequency oscillation in the magnitude and frequency of separated FPSC and FNSC.

4) Analytic relationship between separated sequence components and actual voltage is derived, providing theoretical proof to justify the effectiveness of the designed SMGVO.

5) Voltage-sensorless FCS-MPPC based on extended power theory [22] is implemented with SMGVO and experimentally verified under unbalanced grid condition. Comparisons with conventional SOGI based on voltage sensors was also carried out. Satisfactory performance confirms the effectiveness of the whole control system.

The remainder of this paper is organized as follows. Section II briefly introduces the mathematical model of three phase PWM VSR. Then, the detailed design and analysis of SMGVO is elaborated in Section III. In Section IV, implementation of voltage sensorless FCS-MPPC is introduced. Section V presents experimental results of the whole system to verify the effectiveness of the proposed method. Finally, conclusion is made in Section VI.

\section{Mathematical Equations of PWM VSR}

In the $\alpha \beta 0$ reference frame, the value of all variables can be obtained from the corresponding value in the $a b c$ reference frame using the following Clarke transformation

$$
C_{3 / 2}=\frac{2}{3}\left[\begin{array}{ccc}
1 & -\frac{1}{2} & -\frac{1}{2} \\
0 & \frac{\sqrt{3}}{2} & \frac{\sqrt{3}}{2} \\
\frac{1}{2} & \frac{1}{2} & \frac{1}{2}
\end{array}\right]
$$

The mathematical model of a VSR shown in Fig. 1 can be expressed by complex vectors as

$$
\begin{aligned}
L_{g} \frac{d \boldsymbol{i}_{g}}{d t} & =\boldsymbol{u}_{g}-R_{g} \boldsymbol{i}_{g}-\boldsymbol{u}_{c} \\
& =\left(\boldsymbol{u}_{p}+\boldsymbol{u}_{n}+\boldsymbol{u}_{0}\right)-R_{g} \boldsymbol{i}_{g}-\boldsymbol{u}_{c}
\end{aligned}
$$

where $\boldsymbol{i}_{g}, \boldsymbol{u}_{c}$ and $\boldsymbol{u}_{g}$ are grid current vector, converter voltage vector and grid voltage vector respectively. $\boldsymbol{u}_{p}$ and $\boldsymbol{u}_{n}$ are FPSC and FNSC decomposed from $\boldsymbol{u}_{g} \cdot \boldsymbol{u}_{0}$ is lumped DC offset, which may result from measurement, grid voltage, etc. $L_{g}$ and $R_{g}$ are AC filter's inductance and resistance. 
The traditional power can be calculated based on instantaneous power theory as

$$
\begin{aligned}
P & =\frac{3}{2} \boldsymbol{i}_{g} \odot \boldsymbol{u}_{g} \\
Q & =\frac{3}{2} \boldsymbol{i}_{g} \otimes \boldsymbol{u}_{g}
\end{aligned}
$$

where $\odot$ and $\otimes$ denote dot product and cross product of two complex vectors, respectively. In [22], a new definition of reactive power $Q_{n}$ is used for operation under an unbalanced network. By replacing traditional reactive power $Q$ with $Q_{n}$ in the control algorithm, active power $P$ can be kept constant while maintaining sinusoidal grid currents when grid voltages are unbalanced. $Q_{n}$ can be calculated as

$$
Q_{n}=\frac{3}{2} \boldsymbol{i}_{g} \odot \boldsymbol{u}_{g}^{q}
$$

where $\boldsymbol{u}_{g}^{q}$ is quadrature value of $\boldsymbol{u}_{g}$ and $\boldsymbol{u}_{g}^{q}$ can be expressed as

$$
\boldsymbol{u}_{g}^{q}=-j \boldsymbol{u}_{p}+j \boldsymbol{u}_{n}
$$

It should be noted that $Q_{n}$ is exactly the same as traditional reactive power $Q$ for an ideal grid. During steady state, the derivatives of $\boldsymbol{u}_{p}$ and $\boldsymbol{u}_{n}$ are

$$
\begin{aligned}
\frac{d \boldsymbol{u}_{p}}{d t} & =j \omega_{g} \boldsymbol{u}_{p} \\
\frac{d \boldsymbol{u}_{n}}{d t} & =-j \omega_{g} \boldsymbol{u}_{n}
\end{aligned}
$$

\section{Estimation OF GRID Voltage}

\section{A. Basic Principle of SMGVO}

According to equations (2), (7) and (8), the following SMGVO is designed

$$
\begin{aligned}
L_{g} \frac{d \hat{\boldsymbol{i}}_{g}}{d t} & =\left(\hat{\boldsymbol{u}}_{p}+\hat{\boldsymbol{u}}_{n}+\hat{\boldsymbol{u}}_{0}+\boldsymbol{u}_{s m o}\right)-R_{g} \hat{\boldsymbol{i}}_{g}-\boldsymbol{u}_{c} \\
\frac{d \hat{\boldsymbol{u}}_{p}}{d t} & =j \omega_{g} \hat{\boldsymbol{u}}_{p}+\omega_{c} \boldsymbol{u}_{s m o} \\
\frac{d \hat{\boldsymbol{u}}_{n}}{d t} & =-j \omega_{g} \hat{\boldsymbol{u}}_{n}+\omega_{c} \boldsymbol{u}_{s m o} \\
\frac{d \hat{\boldsymbol{u}}_{0}}{d t} & =\omega_{c 0} \boldsymbol{u}_{s m o}
\end{aligned}
$$

where the hat ${ }^{\wedge}$ represents estimated variable and $\boldsymbol{u}_{s m o}$ is sliding mode control function needs to be designed; $\hat{\boldsymbol{u}}_{0}$ is the estimated DC component which can eliminate DC drift in the estimated FPSC $\hat{\boldsymbol{u}}_{p}$ and FNSC $\hat{\boldsymbol{u}}_{n} . \omega_{c}$ and $\omega_{c 0}$ are positive gains for voltage estimation.

Subtracting (9) from (2) gives the dynamics of current tracking error

$$
L_{g} \frac{d \boldsymbol{e}_{i}}{d t}=\left(\boldsymbol{e}_{p}+\boldsymbol{e}_{n}+\boldsymbol{e}_{0}\right)-R_{g} \boldsymbol{e}_{i}-\boldsymbol{u}_{s m o}
$$

where $\boldsymbol{e}_{i}=\boldsymbol{i}_{g}-\hat{\boldsymbol{i}}_{g}, \boldsymbol{e}_{p}=\boldsymbol{u}_{p}-\hat{\boldsymbol{u}}_{p}, \boldsymbol{e}_{n}=\boldsymbol{u}_{n}-\hat{\boldsymbol{u}}_{n}, \boldsymbol{e}_{0}=$ $\boldsymbol{u}_{0}-\hat{\boldsymbol{u}}_{0}$ are estimation errors of grid current, FPSC, FNSC and DC component respectively. In this paper, sliding surface is chosen as current tracking error, i.e.,

$$
S=e_{i}=i_{g}-\hat{i}_{g}
$$

For better performance, a constant plus proportional rate reaching law [28]

$$
\frac{d \boldsymbol{S}}{d t}=-h \cdot \operatorname{sgn}(\boldsymbol{S})-\lambda \cdot \boldsymbol{S}
$$

is employed on the design of $\boldsymbol{u}_{s m o} . h$ and $\lambda$ are reaching law parameters. For a complex vector, switching function $\operatorname{sgn}(\boldsymbol{S})$ is defined as

$$
\operatorname{sgn}(\boldsymbol{S})=\frac{\boldsymbol{S}}{|\boldsymbol{S}|}
$$

According to (14), inserting (15) into (13) yields the following equation

$$
L_{g} h \cdot \operatorname{sgn}(\boldsymbol{S})+L_{g} \lambda \cdot \boldsymbol{S}=R_{g} \cdot \boldsymbol{S}-\left(\boldsymbol{e}_{p}+\boldsymbol{e}_{0}+\boldsymbol{e}_{n}\right)+\boldsymbol{u}_{s m o} .
$$

Based on (17), $\boldsymbol{u}_{s m o}$ can be calculated according to (18) with voltage tracking errors considered as disturbance.

$$
\boldsymbol{u}_{s m o}=L_{g} h \cdot \operatorname{sgn}(\boldsymbol{S})+\left(L_{g} \lambda-R_{g}\right) \boldsymbol{S} .
$$

\section{B. Stability and Performance Analysis of SMGVO}

To evaluate stability of the designed SMGVO, the following Lyapunov function is defined.

$$
V=\frac{|\boldsymbol{S}|^{2}}{2}=\frac{S_{\alpha}^{2}+S_{\beta}^{2}}{2} .
$$

The time derivative of $V$ is

$$
\dot{V}=\dot{S}_{\alpha} S_{\alpha}+\dot{S}_{\beta} S_{\beta}=\dot{\boldsymbol{S}} \odot \boldsymbol{S} .
$$

The estimation error of current $\boldsymbol{e}_{i}$ will always move to zero if

$$
\dot{V}=\dot{\boldsymbol{S}} \odot \boldsymbol{S}=\dot{\boldsymbol{e}}_{i} \odot \boldsymbol{e}_{i}<0 .
$$

According to (13) and (18), condition (21) can be rearranged as

$$
\left(\boldsymbol{e}_{p}+\boldsymbol{e}_{n}+\boldsymbol{e}_{0}\right) \odot \boldsymbol{e}_{i}-L_{g} h\left|\boldsymbol{e}_{i}\right|-L_{g} \lambda\left|\boldsymbol{e}_{i}\right|^{2}<0
$$

Considering

$$
\left(\boldsymbol{e}_{p}+\boldsymbol{e}_{n}+\boldsymbol{e}_{0}\right) \odot \boldsymbol{e}_{i}<\left|\boldsymbol{e}_{p}+\boldsymbol{e}_{n}+\boldsymbol{e}_{0}\right| \cdot\left|\boldsymbol{e}_{i}\right|
$$

$k$ and $\lambda$ can be chosen as

$$
\begin{aligned}
& h>\frac{\left|\boldsymbol{e}_{p}+\boldsymbol{e}_{n}+\boldsymbol{e}_{0}\right|}{L_{g}} \\
& \lambda>0
\end{aligned}
$$

to ensure the state variable can always approach the sliding surface.

According to (13) and (18), the following transfer function can be obtained.

$$
F_{e}(s)=\frac{\boldsymbol{e}_{i}(s)}{\boldsymbol{e}_{u}(s)}=\frac{1}{L_{g}} \frac{1}{s+h /\left|\boldsymbol{e}_{i}\right|+\lambda}
$$

where, $\boldsymbol{e}_{u}=\boldsymbol{e}_{p}+\boldsymbol{e}_{n}+\boldsymbol{e}_{0}$ is the lumped estimation errors of voltages. It can be seen from (26) that the minimum bandwidth of $F_{e}$ is $\lambda$. Hence, a larger $\lambda$ can result in faster dynamic response. Additionally, a larger $h$ is required to increase robustness against estimation errors of voltages as can be seen from (24). But on the other hand, smaller values of $h$ and $\lambda$ are preferable in practical application for better noise immunity. In this paper, they are set as $h=2000$ and $\lambda=1000$ for a 


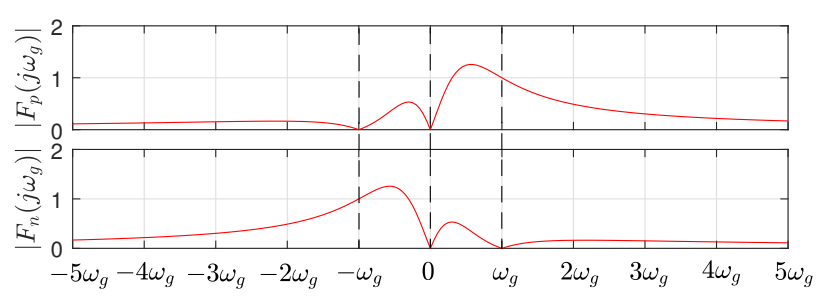

Fig. 2. Amplitude-frequency characteristic of $F_{p}(s)$ and $F_{n}(s)$ with $\omega_{c}=$ $0.707 \omega_{g}$ and $\omega_{c 0}=0.2 \omega_{g}$.

tradeoff between dynamic and steady-state performance based on experimental tests.

Though satisfactory performance of SMGVO can be guaranteed by properly selected parameters, the relationship between estimated value and actual voltage is still unclear. Since $\boldsymbol{S}=0$ when moving along sliding surface, (17) can be simplified as

$$
\begin{aligned}
\boldsymbol{u}_{s m o} & =\left(\boldsymbol{e}_{p}+\boldsymbol{e}_{n}+\boldsymbol{e}_{0}\right) \\
& =\boldsymbol{u}_{g}-\hat{\boldsymbol{u}}_{p}-\hat{\boldsymbol{u}}_{n}-\hat{\boldsymbol{u}}_{0} .
\end{aligned}
$$

Based on (10)-(12) and (27), the relationship between estimated components and $\boldsymbol{u}_{g}$ can be described by the following transfer functions when moving along sliding surface:

$$
\begin{aligned}
& F_{p}(s)=\frac{\hat{\boldsymbol{u}}_{p}(s)}{\boldsymbol{u}_{g}(s)}=\frac{\omega_{c} s\left(s+j \omega_{g}\right)}{s^{3}+\left(\omega_{c 0}+2 \omega_{c}\right) s^{2}+\omega_{g}^{2} s+\omega_{c 0} \omega_{g}^{2}} \\
& F_{n}(s)=\frac{\hat{\boldsymbol{u}}_{n}(s)}{\boldsymbol{u}_{g}(s)}=\frac{\omega_{c} s\left(s-j \omega_{g}\right)}{s^{3}+\left(\omega_{c 0}+2 \omega_{c}\right) s^{2}+\omega_{g}^{2} s+\omega_{c 0} \omega_{g}^{2}}
\end{aligned}
$$

It can be seen from $F_{p}(s)$ and $F_{n}(s)$ that two third-order complex coefficient filters are implicitly incorporated in the designed SMGVO. Based on some simple calculations, one can find that

$$
\begin{aligned}
& \begin{cases}F_{p}\left(j \omega_{g}\right) & =1 \\
F_{p}\left(-j \omega_{g}\right) & =0, \\
F_{p}(0) & =0\end{cases} \\
& \begin{cases}F_{n}\left(j \omega_{g}\right) & =0 \\
F_{n}\left(-j \omega_{g}\right) & =1 . \\
F_{n}(0) & =0\end{cases}
\end{aligned}
$$

Fig. 2 shows the amplitude-frequency characteristic of $F_{p}(s)$ and $F_{n}(s)$. It is clear that $F_{p}(s)$ has unity gain at the fundamental positive frequency (FPF) and a zero gain at both fundamental negative frequency (FNF) and zero frequency. While $F_{n}(s)$ presents unity gain at FNF and zero gain at both FPF and zero frequency. Obviously, the designed SMGVO can accurately separate FPSC and FNSC without the DC component. Additionally, both $F_{p}(s)$ and $F_{n}(s)$ show filtering effect on high frequency harmonics. Hence, high frequency chattering can be well suppressed.
If $\omega_{c 0}$ in (12) is set as zero, namely estimation of DC component is not considered, (28) and (29) would be two second-order complex filters as follows

$$
\begin{aligned}
& F_{p 1}(s)=\frac{\hat{\boldsymbol{u}}_{p}(s)}{\boldsymbol{u}_{g}(s)}=\frac{\omega_{c}\left(s+j \omega_{g}\right)}{s^{2}+2 \omega_{c} s+\omega_{g}^{2}}, \\
& F_{n 1}(s)=\frac{\hat{\boldsymbol{u}}_{n}(s)}{\boldsymbol{u}_{g}(s)}=\frac{\omega_{c}\left(s-j \omega_{g}\right)}{s^{2}+2 \omega_{c} s+\omega_{g}^{2}} .
\end{aligned}
$$

By setting $\omega_{c}=0.707 \omega_{g}$, satisfactory dynamic performance and steady-state performance can be obtained [29]. To reduce the interference between DC component and estimations of FPSC and FNSC, $\omega_{c 0}$ is set as a smaller value $\omega_{c 0}=0.2 \omega_{g}$ in this paper.

\section{Effects of Parameter Mismatches}

Considering that the estimated inductance and resistance used in the observer are $\hat{L}_{g}$ and $\hat{R}_{g}$, parameter errors can then be defined as

$$
\Delta L=L_{g}-\hat{L}_{g}, \Delta R=R_{g}-\hat{R}_{g}
$$

Following the same procedure as deriving (28) and (29), the estimated FPSC and FNSC become

$$
\begin{aligned}
& \hat{\boldsymbol{u}}_{p}(s)=F_{p}(s)\left(\boldsymbol{u}_{g}(s)-\Delta L \cdot s \boldsymbol{i}_{g}(s)-\Delta R \boldsymbol{i}_{g}(s)\right) \\
& \hat{\boldsymbol{u}}_{n}(s)=F_{n}(s)\left(\boldsymbol{u}_{g}(s)-\Delta L \cdot s \boldsymbol{i}_{g}(s)-\Delta R \boldsymbol{i}_{g}(s)\right)
\end{aligned}
$$

As $F_{p}(s)$ can selectively pass FPSC and isolate FNSC, the relationship between estimated $\hat{\boldsymbol{u}}_{p}$ and its actual value $\boldsymbol{u}_{p}$ can be derived during steady state as

$$
\hat{\boldsymbol{u}}_{p}=\boldsymbol{u}_{p}-j \omega_{g} \Delta L \boldsymbol{i}_{g p}-\Delta R \boldsymbol{i}_{g p}
$$

where $\boldsymbol{i}_{g p}$ is the FPSC of grid current $\boldsymbol{i}_{g}$. Similarly, the relationship between $\hat{\boldsymbol{u}}_{n}$ and $\boldsymbol{u}_{n}$ can be obtained as

$$
\hat{\boldsymbol{u}}_{n}=\boldsymbol{u}_{n}+j \omega_{g} \Delta L \boldsymbol{i}_{g n}-\Delta R \boldsymbol{i}_{g n}
$$

From (37) and (38), one can see that parameter mismatches would introduce estimation errors in both $\hat{\boldsymbol{u}}_{p}$ and $\hat{\boldsymbol{u}}_{n}$. The deviation depends on parameter mismatches and actual current. However, due to selective ability of $F_{p}(s)$ and $F_{n}(s)$, $\hat{\boldsymbol{u}}_{p}$ still only contains positive-sequence component and $\hat{\boldsymbol{u}}_{n}$ still only contains negative-sequence component. With system parameters shown in Table. I, simulation results of magnitude and phase errors of $\hat{\boldsymbol{u}}_{p}$ and $\hat{\boldsymbol{u}}_{n}$ are illustrated in Figs. 3 and 4. It can be found that inductance mismatch has less effect on magnitude error than that caused by resistance mismatch. However, inductance error dominates estimation errors of phase angle. Assuming model parameters are within twice of actual value, the maximum magnitude error is about $4 \%$ of actual value for $\hat{\boldsymbol{u}}_{p}$ and $\hat{\boldsymbol{u}}_{n}$. And, the maximum phase error is about $0.2 \mathrm{rad}$. 


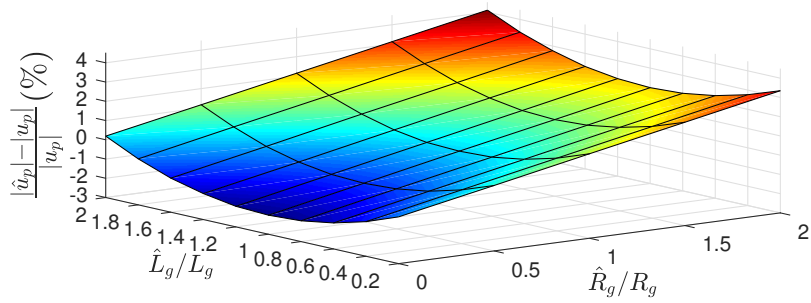

(a)

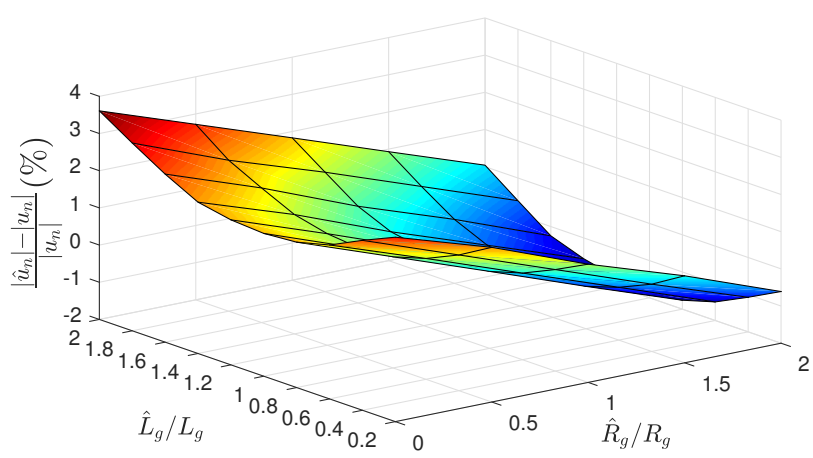

(b)

Fig. 3. Simulated magnitude error of (a) $\hat{\boldsymbol{u}}_{p}$ and (b) $\hat{\boldsymbol{u}}_{n}$ when $\hat{L}_{g}$ varies from $0.2 L_{g}$ to $2 L_{g}$ and $\hat{R}_{g}$ varies from 0 to $2 R_{g}$.

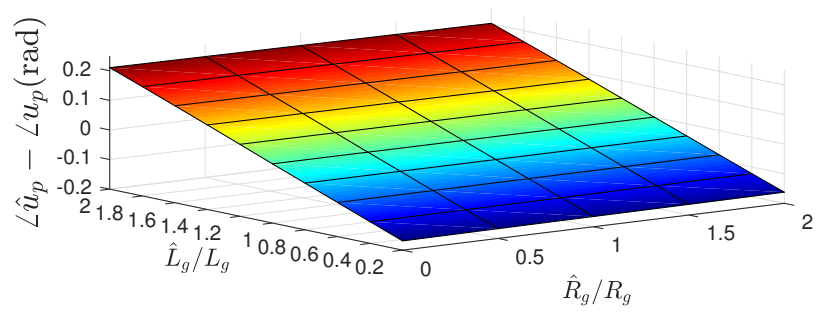

(a)

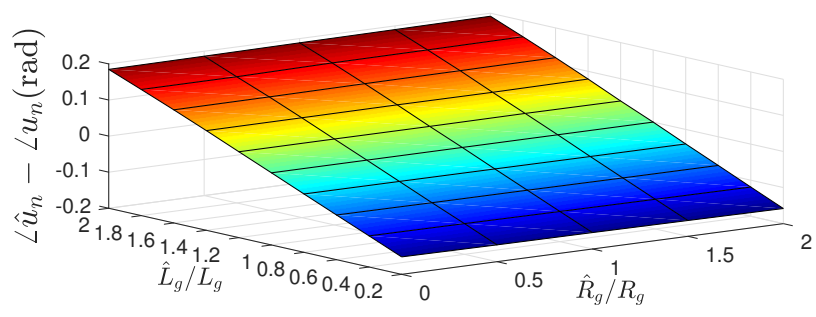

(b)

Fig. 4. Simulated phase error of (a) $\hat{\boldsymbol{u}}_{p}$ and (b) $\hat{\boldsymbol{u}}_{n}$ when $\hat{L}_{g}$ varies from $0.2 L_{g}$ to $2 L_{g}$ and $\hat{R}_{g}$ varies from 0 to $2 R_{g}$.

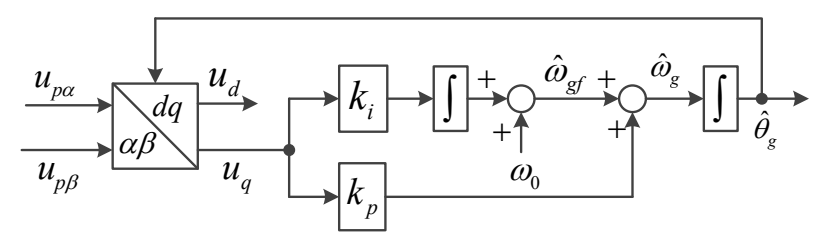

Fig. 5. Block diagram of SRF-PLL.

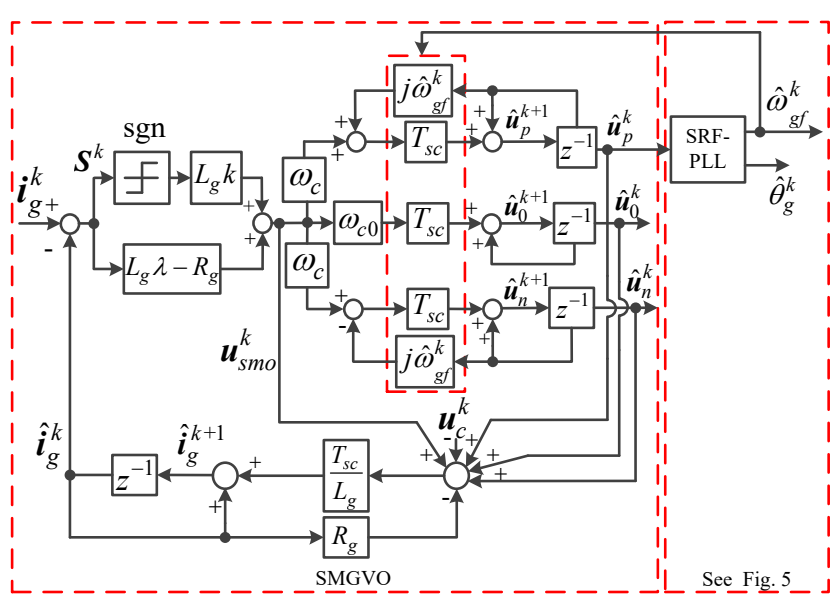

Fig. 6. Block diagram of the proposed SMGVO.

\section{Implementation of SMGVO}

With a small sampling period $T_{s c}$, the simple first-order Euler discretization method can be employed to rewrite (9)(12) in the discrete-time form as

$$
\begin{aligned}
& \boldsymbol{u}_{s m o}^{k}=L_{g} k \cdot \operatorname{sgn}\left(\boldsymbol{i}_{g}^{k}-\hat{\boldsymbol{i}}_{g}^{k}\right)+\left(L_{g} \lambda-R_{g}\right)\left(\boldsymbol{i}_{g}^{k}-\hat{\boldsymbol{i}}_{g}^{k}\right) \\
& \hat{\boldsymbol{i}}_{g}^{k+1}=\hat{\boldsymbol{i}}_{g}^{k}+\frac{T_{s c}}{L_{g}}\left[\hat{\boldsymbol{u}}_{p}^{k}+\hat{\boldsymbol{u}}_{n}^{k}+\hat{\boldsymbol{u}}_{0}^{k}+\boldsymbol{u}_{s m o}^{k}-R_{g} \hat{\boldsymbol{i}}_{g}^{k}-\boldsymbol{u}_{c}^{k}\right] \\
& \hat{\boldsymbol{u}}_{p}^{k+1}=\hat{\boldsymbol{u}}_{p}^{k}+T_{s c}\left(\omega_{c} \boldsymbol{u}_{s m o}^{k}+j \omega_{g} \hat{\boldsymbol{u}}_{p}^{k}\right) \\
& \hat{\boldsymbol{u}}_{n}^{k+1}=\hat{\boldsymbol{u}}_{n}^{k}+T_{s c}\left(\omega_{c} \boldsymbol{u}_{s m o}^{k}-j \omega_{g} \hat{\boldsymbol{u}}_{n}^{k}\right) \\
& \hat{\boldsymbol{u}}_{0}^{k+1}=\hat{\boldsymbol{u}}_{0}^{k}+T_{s c} \omega_{c 0} \boldsymbol{u}_{s m o}^{k}
\end{aligned}
$$

It should be noted that no explicit filters are utilized here. However, two implicit complex coefficient filters, i.e., (28) and (29) are inherent filtering characteristics of the designed SMGVO. Hence, FPSC and FNSC can be accurately estimated and separated while suppressing the influence of DC offset. As $\hat{\boldsymbol{u}}_{p}$ and $\hat{\boldsymbol{u}}_{n}$ are obtained by integrating $\boldsymbol{u}_{\text {smo }}$, chattering and sampling noises included in $\boldsymbol{u}_{\text {smo }}$ are suppressed due to the filtering effect of the integrator. This can also be confirmed by amplitude-frequency characteristic shown in Fig. 2.

As seen from (41) and (42), actual grid frequency $\omega_{g}$ is required for the proposed SMGVO to estimate FPSC and FNSC. To obtain better estimation accuracy under grid frequency variation, the synchronous reference frame PLL (SRF-PLL) as shown in Fig. 5 can be employed for online frequency adaption. In the figure, the input voltages $u_{p \alpha}$ and $u_{p \beta}$ are normalized scalar components of $\hat{\boldsymbol{u}}_{p}^{k}$, i.e.,

$$
u_{p \alpha}=\frac{\hat{u}_{p \alpha}^{k}}{\left|\hat{\boldsymbol{u}}_{p}^{k}\right|}, u_{p \beta}=\frac{\hat{u}_{p \beta}^{k}}{\left|\hat{\boldsymbol{u}}_{p}^{k}\right|} .
$$

After normalization, the linearized model of SRF-PLL can be derived as [12]

$$
G_{\theta}=\frac{\hat{\theta}_{g}(s)}{\theta_{g}(s)}=\frac{k_{p} s+k_{i}}{s^{2}+\underbrace{k_{p}}_{2 \zeta \omega_{n}} s+\underbrace{k_{i}}_{\omega_{n}^{2}}},
$$


where $k_{p}$ and $k_{i}$ are gains of proportional-integral (PI) controller; $\zeta$ and $\omega_{n}$ are damping factor and natural frequency respectively; $k_{p}=2 \zeta \omega_{n}$; and $k_{i}=\omega_{n}^{2}$.

In practical application, actual grid frequency may deviates from its nominal value. To mitigate side-effects of frequency variation on the observer, it is necessary to feed back estimated $\hat{\omega}_{g}$ from SRF-PLL stage to the observer. It is clear that the frequency feedback loop (FFL) consists of two parts (see Fig. 6): inner observer and outer SRF-PLL. To maintain stability, the bandwidth of outer loop should be slower than inner loop. In [30], this is achieved by inserting a low-pass filter between two parts. From Fig. 5, it can be seen that estimated frequency deviation $\Delta \hat{\omega}_{g}$ consists of proportional term $k_{p} u_{q}$ and integral term $k_{i} u_{q} / s$. An inherent filtered estimation can be obtained from integral component in PI as

$$
\Delta \hat{\omega}_{g f}=\frac{k_{i} \cdot u_{q}}{s}
$$

The relationship between $\Delta \hat{\omega}_{g}$ and $\Delta \hat{\omega}_{g f}$ can be easily derived as

$$
G_{\omega}(s)=\frac{\Delta \hat{\omega}_{g f}(s)}{\Delta \hat{\omega}_{g}(s)}=\frac{k_{i}}{k_{p} s+k_{i}}
$$

It can be seen that $G_{\omega}$ is a low pass filter that can reduce interference between SMGVO and SRF-PLL. With $\Delta \hat{\omega}_{g f}$, the filtered grid frequency can be estimated as

$$
\hat{\omega}_{g f}=\omega_{0}+\Delta \hat{\omega}_{g f}
$$

where $\omega_{0}$ is the nominal frequency, which is $2 \pi 50 \mathrm{rad} / \mathrm{s}$ in this paper. As SMGVO based PLL is a highly nonlinear system, it is difficult to design parameters analytically. Though better results can be obtained by deriving small-signal linearized model as performed in [31], parameters can also be tuned by simulation [32]. In this paper, damping factor is set as $\zeta=1$ to reduce overshoot during transient process. $\omega_{n}$ is chosen as $\omega_{n}=2 \pi 15 \mathrm{rad} / \mathrm{s}$ for the compromise between dynamic responses and immunity against noises and harmonics. This gives $k_{p}=188.5$ and $k_{i}=8882.6$. To verify the effectiveness of parameter selection, steady-sate and dynamic performance are experimentally tested in Section V under both unbalanced and distorted conditions. A detailed block diagram of the proposed SMGVO with SRF-PLL is shown in Fig. 6.

In this paper, SMGVO is designed and analyzed in continuous-time domain. After that, it is discretized for practical implementation. With a high sampling frequency, this is usually acceptable and satisfactory performance can be obtained as those presented in [4], [10], [15], [33]. Better performance may be obtained by design and analysis in discretetime domain. However, the derivation process would be more complicated. Nevertheless, if changing rate of the concerned state variable is comparable with sampling frequency, design and analysis in discrete-time domain give better results [3].

\section{SMGVO BASED FCS-MPPC}

In [22], FCS-MPPC employing $Q_{n}$ as shown in (5) is investigated under unbalanced grid conditions. The control diagram of FCS-MPPC based on the proposed SMGVO is shown in Fig. 7. From (39)-(43), it can be found that SMGVO is not only an estimator but also a predictor. The predicted

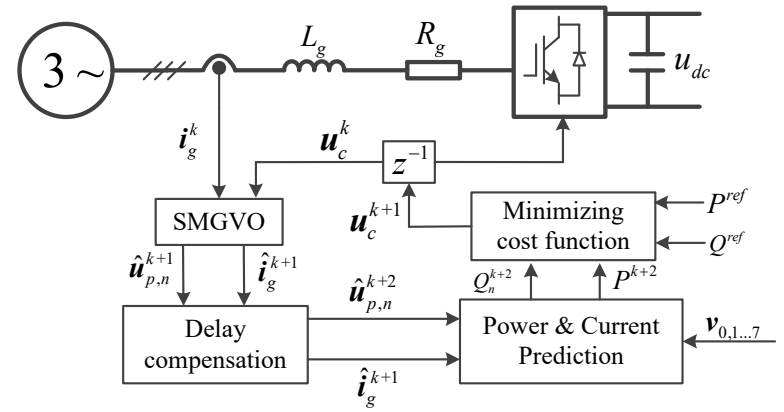

Fig. 7. Control diagram of SMGVO based FCS-MPPC.

TABLE I

CONTROL AND SYSTEM PARAMETERS

\begin{tabular}{lcc}
\hline Filter resistance & $R_{g}$ & $0.3 \Omega$ \\
Filter inductance & $L_{g}$ & $10 \mathrm{mH}$ \\
Line-line voltage (RMS) & $U_{N}$ & $150 \mathrm{~V}$ \\
Voltage frequency & $f$ & $50 \mathrm{~Hz}$ \\
DC-side capacitor & $C$ & $840 \mu \mathrm{F}$ \\
Load resistance & $R_{L}$ & $100 \Omega$ \\
Sampling period & $T_{s c}$ & $50 \mu \mathrm{s}$ \\
SMGVO parameter 1 & $h$ & 2000 \\
SMGVO parameter 2 & $\lambda$ & 1000 \\
SMGVO parameter 3 & $\omega_{c}$ & $0.707 \omega_{g}$ \\
SMGVO parameter 4 & $\omega_{c 0}$ & $0.2 \omega_{g}$ \\
\hline
\end{tabular}

current and voltage at $(k+1)$ th instant can be utilized to compensate for the one-step delay in practical application. The detailed implementation steps of FCS-MPPC based on SMGVO can be summarized as follows:

1) Calculating $\hat{\boldsymbol{i}}_{g}^{k+1}, \hat{\boldsymbol{u}}_{p}^{k+1}, \hat{\boldsymbol{u}}_{n}^{k+1}$ and $\hat{\boldsymbol{u}}_{0}^{k+1}$ based on (39)(43).

2) Predicting $\hat{\boldsymbol{u}}_{p}^{k+2}$ and $\hat{\boldsymbol{u}}_{n}^{k+2}$ as

$$
\left\{\begin{array}{l}
\hat{\boldsymbol{u}}_{p}^{k+2}=\hat{\boldsymbol{u}}_{p}^{k+1} e^{j \omega_{g} T_{s c}} \\
\hat{\boldsymbol{u}}_{n}^{k+2}=\hat{\boldsymbol{u}}_{n}^{k+1} e^{-j \omega_{g} T_{s c}}
\end{array} .\right.
$$

3) Grid current $\boldsymbol{i}_{g m}^{k+2}$ for all candidate voltage vectors $\boldsymbol{v}_{m}(m=0,1 \ldots 7)$ can be predicted based on (2) with estimated grid voltages and current in step 1) as

$$
\begin{aligned}
\boldsymbol{i}_{g m}^{k+2}=\hat{\boldsymbol{i}}_{g}^{k+1}+\frac{T_{s c}}{L_{g}}\left[\hat{\boldsymbol{u}}_{p}^{k+1}+\right. & \hat{\boldsymbol{u}}_{n}^{k+1}+\hat{\boldsymbol{u}}_{0}^{k+1} \\
& \left.-R_{g} \hat{\boldsymbol{i}}_{g}^{k+1}-\boldsymbol{v}_{m}\right]
\end{aligned}
$$

4) Predicting active power $P_{m}^{k+2}$ and reactive power $Q_{n, m}^{k+2}$ for all calculated $i_{g m}^{k+2}$ based on (3), (5) and (6) as

$$
\begin{aligned}
P_{m}^{k+2} & =\frac{3}{2} \boldsymbol{i}_{g m}^{k+2} \odot\left(\hat{\boldsymbol{u}}_{p}^{k+2}+\hat{\boldsymbol{u}}_{n}^{k+2}\right), \\
Q_{n, m}^{k+2} & =\frac{3}{2} \boldsymbol{i}_{g m}^{k+2} \odot\left(j \hat{\boldsymbol{u}}_{n}^{k+2}-j \hat{\boldsymbol{u}}_{p}^{k+2}\right) .
\end{aligned}
$$

5) Evaluating the following cost function

$$
\begin{gathered}
J_{m}=\left|P^{\text {ref }}-P_{m}^{k+2}\right|^{2}+\left|Q^{r e f}-Q_{n, m}^{k+2}\right|^{2}+I_{C} \\
I_{c}= \begin{cases}10^{8}+10^{8}\left(\left|i_{g m}^{k+2}\right|-I_{\text {max }}\right) & \text { if }\left|i_{g m}^{k+2}\right|>I_{\text {max }} \\
0 & \text { if }\left|i_{g m}^{k+2}\right|<I_{\text {max }}\end{cases}
\end{gathered}
$$




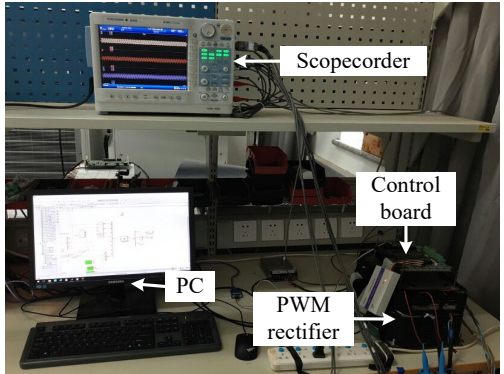

(a)

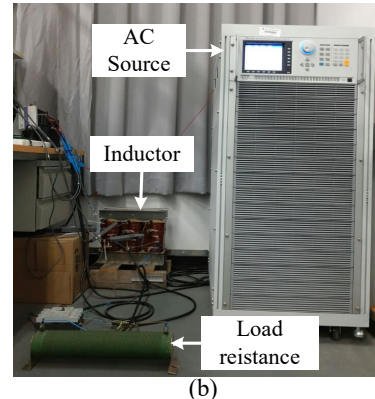

(b)
Fig. 8. Experimental test setup.

The voltage vector $\boldsymbol{v}_{m}$ which leads to minimum value of $J_{m}$ is selected as the output voltage of the converter.

In (54), $I_{c}$ is added in the cost function to avoid over current during transient process [33]. For experimental tests in this paper, $I_{\max }$ is set as $8 \mathrm{~A}$. The reason why $Q_{n}$ is more suitable for power control under unbalanced grid condition has been explained in [22] and thus not repeated here.

It should be noted that if voltage sensors are equipped, FPSC and FNSC still need to be separated using methods such as SOGI [34] or delayed signal cancellation filter [12]. Additionally, equations (40)-(42) should also be implemented to compensate one-step control delay with measured values. In the designed SMGVO, estimation and prediction are integrated into one stage. Not only can FPSC and FNSC be obtained but also predictions of current and voltages at $(k+1)$ th instant are directly available for delay compensation. Therefore, SMGVO based voltage sensorless predictive power control would not increase much in complexity compared with conventional voltage sensors based predictive control schemes.

\section{EXPERIMENTAL RESULTS}

The performance of the designed control system is evaluated on a laboratory test-rig, which is shown in Fig. 8. In all tests, measured grid voltages are not used in the controller but only displayed for comparisons. The experimental data is acquired by a scopecorder DL850. Grid voltages and currents are directly measured by probes, while estimated voltages are obtained via an on-board digital-to-analog converter (DAC7724U). A programmable AC source (Chroma 61511) is used to simulate unbalanced grid conditions. The sampling time is $T_{s c}=50 \mu \mathrm{s}$, other control and system parameters are listed in Table I. In all the following tests, only the performance of power control and voltage estimation is considered and outer DC voltage control loop is not discussed here. For comparison, FPSC and FNSC, i.e., $\boldsymbol{u}_{p}^{\text {sogi }}$ and $\boldsymbol{u}_{n}^{\text {sogi }}$ are calculated from measured grid voltages based on dual SOGI (DSOGI) as follow [29]

$$
\left\{\begin{array}{l}
\boldsymbol{u}_{s g}=\frac{m \omega_{g} s}{s^{2}+m \omega_{g} s+\omega_{g}^{2}} \boldsymbol{u}_{g} \\
\boldsymbol{u}_{s g}^{q}=\frac{m \omega_{g}^{2}}{s^{2}+m \omega_{g} s+\omega_{g}^{2}} \boldsymbol{u}_{g} \\
\boldsymbol{u}_{p}^{s g i}=\frac{1}{2}\left(\boldsymbol{u}_{s g}+j \boldsymbol{u}_{s g}^{q}\right) \\
\boldsymbol{u}_{n}^{s o g i}=\frac{1}{2}\left(\boldsymbol{u}_{s g}-j \boldsymbol{u}_{s g}^{q}\right)
\end{array}\right.
$$

where $m$ is selected as $\sqrt{2}$ for satisfactory dynamic and steady-state performance [29], [34]. The effectiveness of

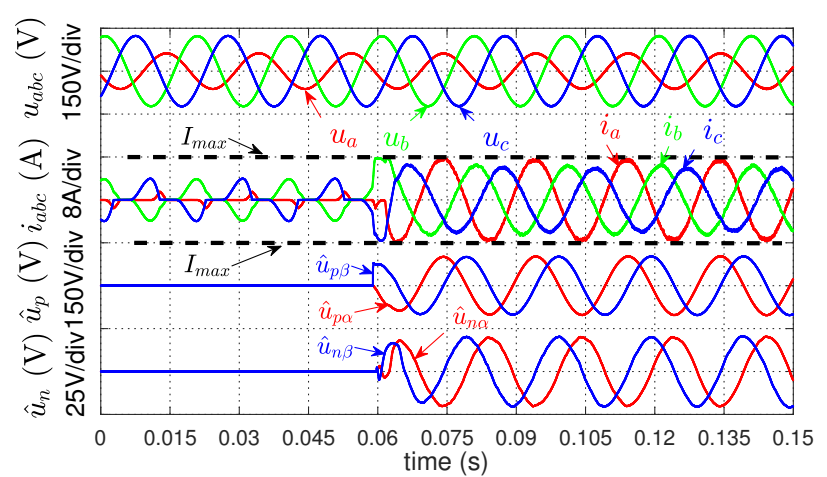

(a)

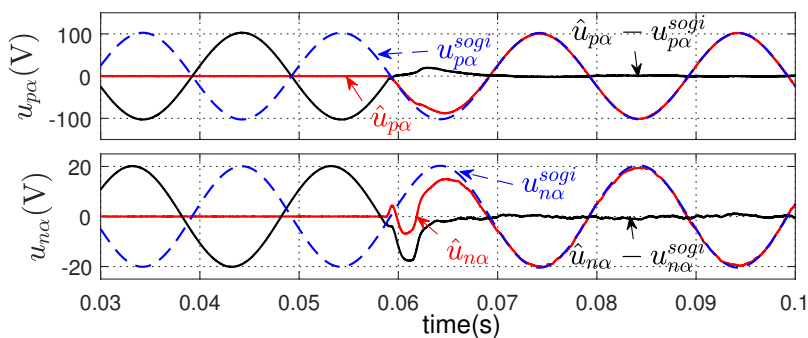

(b)

Fig. 9. Startup responses with $50 \%$ voltage dip in phase A. (a) Actual grid voltages, currents and estimated voltages; (b) comparison between estimated voltages from SMGVO and calculated voltages from DSOGI.

DSOGI has been extensively verified in existing research under unbalanced grid conditions. Additionally, $\boldsymbol{u}_{p}^{\text {sogi }}$ and $\boldsymbol{u}_{n}^{\text {sogi }}$ are directly calculated based on actual voltages sampled by scopecorder. Hence, it is assumed that $\boldsymbol{u}_{p}^{\text {sogi }}$ and $\boldsymbol{u}_{n}^{\text {sogi }}$ are accurate and can be used as the benchmark to verify the effectiveness of the designed SMGVO.

Fig. 9 shows responses from startup process to steady state operation with $50 \%$ voltage dip in phase A. The converter initially works in diode rectifier mode. After a moment, the control system is enabled and then the converter works in PWM rectifier mode. The references of active power $P^{r e f}$ and reactive power $Q^{r e f}$ are set as $1 \mathrm{~kW}$ and 0 Var respectively. It can be seen that SMGVO based FCS-MPPC can safely start without initial knowledge of actual grid voltage. Due to the incorporation of current constraints (54) in the cost function, the magnitude of grid current can be well limited below the $I_{\max }$ during startup process. The waveforms of estimated FPSC $\hat{\boldsymbol{u}}_{p}$ and FNSC $\hat{\boldsymbol{u}}_{n}$ are sinusoidal without obvious chattering. From Fig. 9(b), it is clear that the estimated FPSC and FNSC are in accordance with those extracted by DSOGI from measured grid voltage. The estimation error is small and nearly zero at steady-state. This test confirms that SMGVO can accurately estimate and separate FPSC and FNSC.

To evaluate dynamic responses of SMGVO, a test changing grid voltages from balanced to unbalanced conditions was carried out. 50\% voltage dip in phase A is suddenly applied during normal operation. $P^{r e f}$ and $Q^{r e f}$ are $1 \mathrm{~kW}$ and 0 Var respectively. The results are listed in Fig. 10. It can be seen that estimated FNSC $\hat{\boldsymbol{u}}_{n}$ is nearly zero under balanced condition. When voltage sag is suddenly applied, $\hat{\boldsymbol{u}}_{n}$ can quickly reach its 


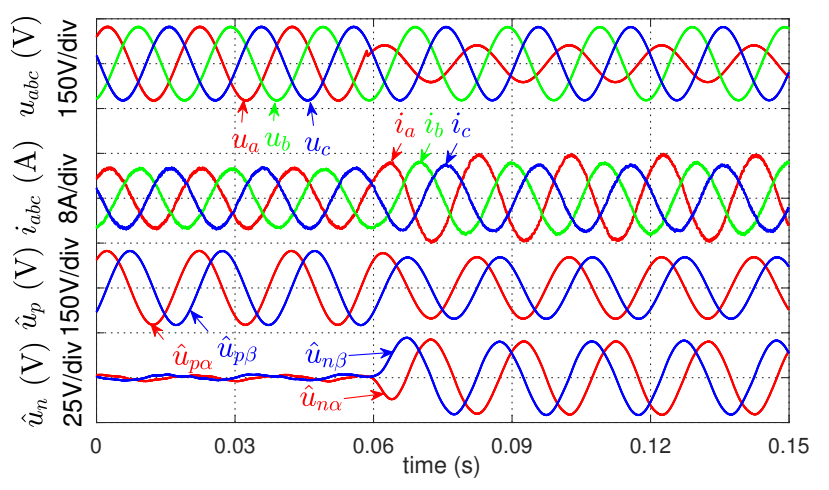

(a)

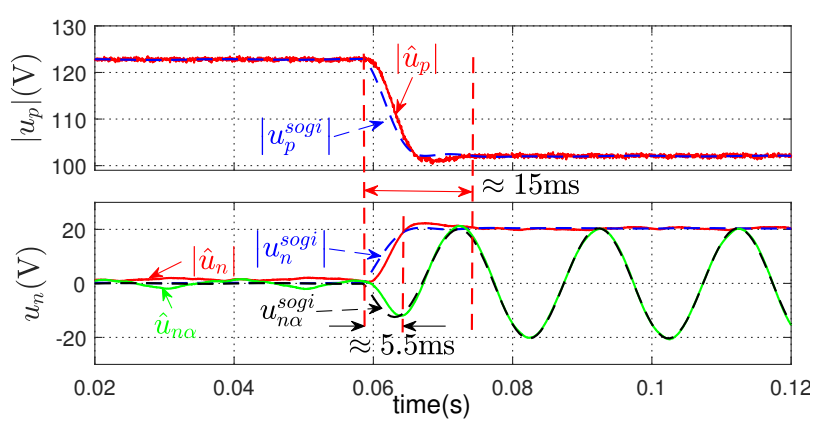

(b)

Fig. 10. Operation from balanced condition to unbalanced condition. (a) Actual grid voltages, currents and estimated voltages; (b) comparison with $\boldsymbol{u}_{p}^{\text {sogi }}$ and $\boldsymbol{u}_{n}^{\text {sogi }}$ calculated from actual voltage by DSOGI.

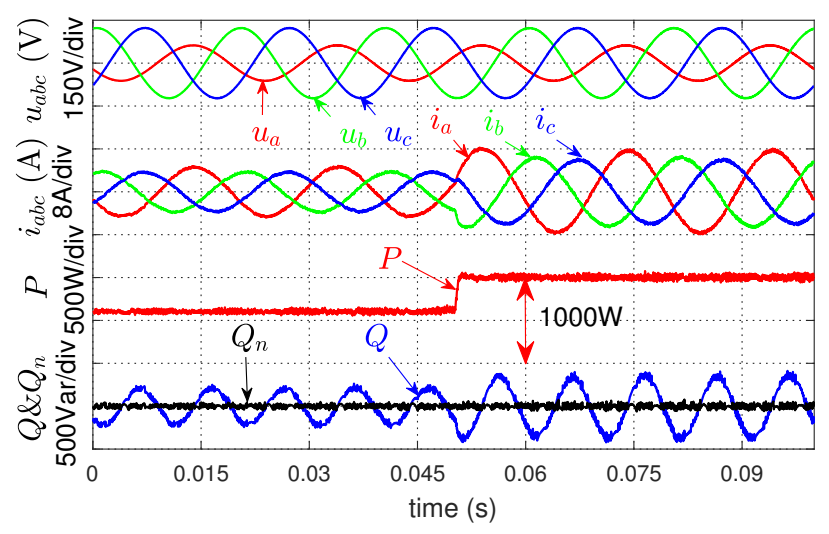

Fig. 11. Dynamic responses when $P^{r e f}$ steps from $600 \mathrm{~W}$ to $1000 \mathrm{~W}$.

steady-state value which is in accordance with that extracted by DSOGI, as can be seen from Fig. 10(b). The rise time of $\hat{\boldsymbol{u}}_{n}$ is about $5.5 \mathrm{~ms}$. For both SMGVO and DSOGI, $2 \%$ settling time is about $15 \mathrm{~ms}$. This test shows that the proposed SMGVO performs well during transient process and its performance is comparable with conventional DSOGI with slightly larger overshoot. Hence, similar performance without significant degradation can be obtained based on SMGVO when compared with voltage-sensors based schemes under unbalanced grid conditions.

Fig. 11 shows dynamic responses when $P^{r e f}$ steps from 600 $\mathrm{W}$ to $1 \mathrm{~kW}$ under unbalanced grid conditions. In the figure, $P$ and $Q$ are calculated according to estimated voltages and measured grid currents based on (3) and (4). $Q_{n}$ is calculated

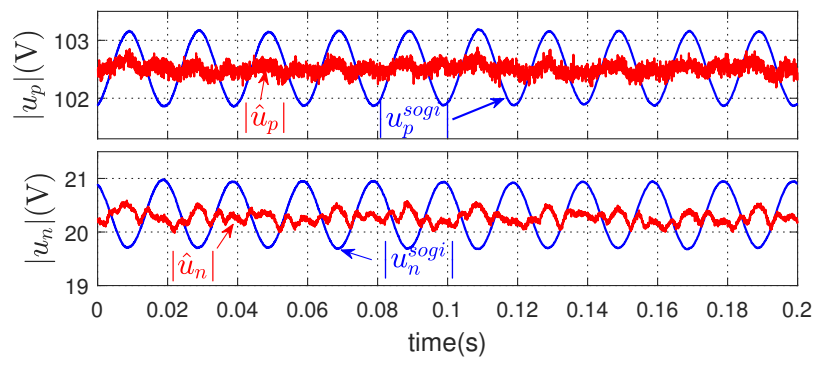

Fig. 12. Steady state responses with $1.5 \mathrm{~V}$ DC component and $50 \%$ AC voltage dip in phase A.

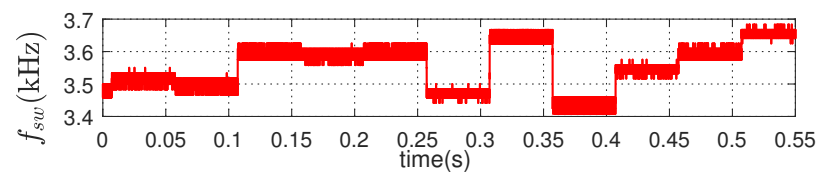

Fig. 13. Average switching frequency $f_{s w}$ when $P^{r e f}=1 \mathrm{~kW}$.

from (5). It can be seen that, obvious twice grid-frequency oscillation exists in conventional reactive power $Q$. However, active power $P$ and new reactive power $Q_{n}$ are constant during steady state and the waveform of grid current is sinusoidal. The obtained results are similar to that of voltage sensors based FCS-MPPC presented in [22]. Additionally, dynamic response is fast without obvious overshoot. During transient process, $Q_{n}$ is kept at zero. Hence, decoupled control of active power and reactive power is achieved. The settling time is less than $3 \mathrm{~ms}$ in this test. The demonstrated results show that SMGVO based voltage sensorless FCS-MPPC could achieve satisfactory performance under unbalanced grid conditions.

A benefit of the proposed SMGVO is that it can eliminate DC component in the estimation. Fig. 12 shows comparative results of SMGVO and DSOGI when there is $1.5 \mathrm{~V}$ DC offset in Phase A under unbalanced grid conditions. It can be seen that significant fundamental frequency oscillations caused by DC component exist in $\boldsymbol{u}_{p}^{s o g i}$ and $\boldsymbol{u}_{n}^{\text {sogi }}$. This is because $\boldsymbol{u}_{s g}^{q}$ in (55) has nonzero gain at zero frequency [8]. While the amplitudes of estimated $\hat{\boldsymbol{u}}_{p}$ and $\hat{\boldsymbol{u}}_{n}$ are much less influenced by injected DC offset.

Fig. 13 shows switching frequency with $P^{r e f}=1 \mathrm{~kW}$ during steady-state operation. In the experimental test, $f_{s w}$ is calculated by counting total switching jumps $N$ of all six legs of PWM rectifier over a fixed period of 0.05 s, i.e., $f_{s w}=N / 6 / 0.05$. Hence, $f_{s w}$ is updated with a rate of $0.05 \mathrm{~s}$ in this test. The average switching frequency over the time span shown in Fig. 13 is about $3.56 \mathrm{kHz}$.

To further evaluate the quality of voltage estimation, the total harmonic distortion (THD) of $\hat{\boldsymbol{u}}_{p}$ and $\hat{\boldsymbol{u}}_{n}$ are calculated when grid voltages are both unbalanced and distorted. In this test, both $14 \mathrm{~V}(\approx 11 \%$ of fundamental value $)-5$ th and 7 th harmonics are injected into grid voltages. Meanwhile, 50\% voltage dip in phase A is applied. Though harmonics cannot be completely removed in the estimation, they are significantly attenuated. The results confirm that the proposed observer can effectively suppress harmonics while estimating fundamental component, which is in accordance with the analysis presented 


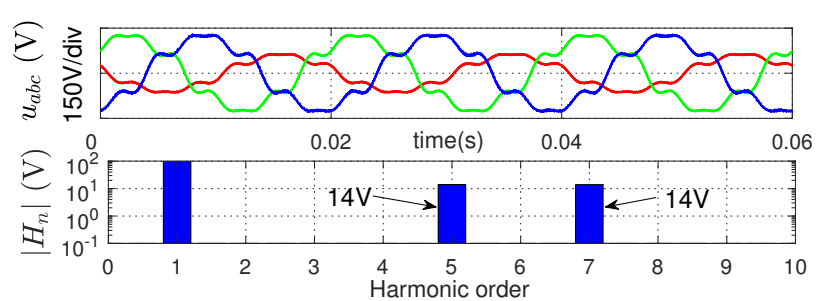

(a)

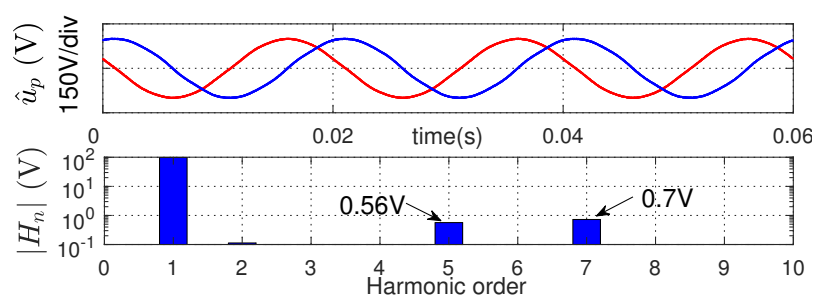

(b)

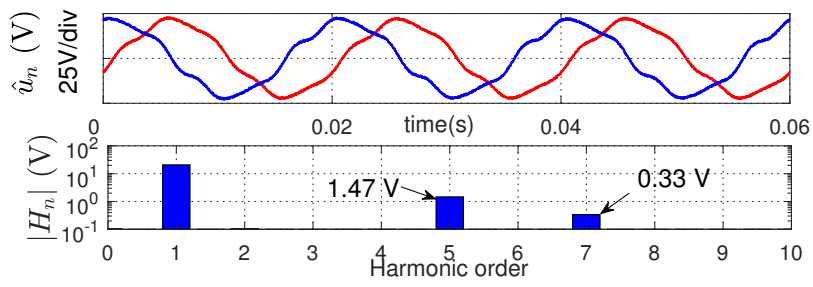

(c)

Fig. 14. Spectrum analysis of (a) grid voltage, (b) $\hat{u}_{p}$ and (b) $\hat{u}_{n}$ under unbalanced and distorted grid conditions.

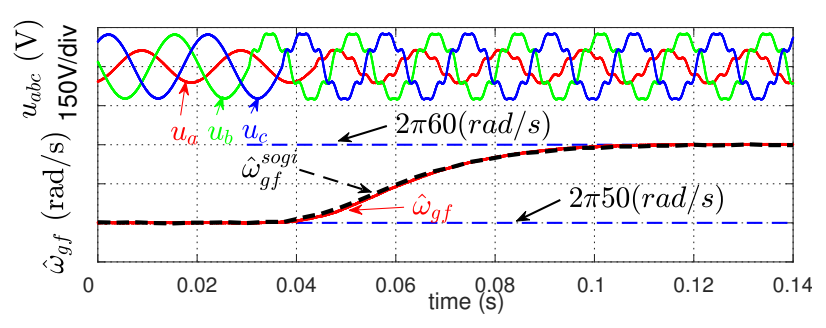

Fig. 15. Estimated grid frequency with sudden frequency step change of +10 $\mathrm{Hz}$ under unbalanced and distorted grid conditions.

\section{in Section III-B.}

Fig. 15 shows the curve of estimated grid frequency when a sudden frequency change of $+10 \mathrm{~Hz}$ is applied. Meanwhile, -5 th and 7 th harmonics are injected into grid voltages. For comparison, $\hat{\omega}_{g f}^{\text {sogi }}$ is obtained by replacing $\hat{\boldsymbol{u}}_{p}$ with $\boldsymbol{u}_{p}^{\text {sogi }}$ as the input voltage of SRF-PLL. It is seen that with SRFPLL as shown in Fig. 5, the designed SMGVO can be adapted to frequency variation. Both dynamic and steady-state performance are similar with that obtained from conventional DSOGI. Due to the ability to suppress harmonics, estimated grid frequency is smooth without significant oscillation even under distorted grid conditions.

\section{CONCLUSION}

A SMGVO is designed and experimentally verified in this paper. It has the following properties: 1) inherent separation of FPSC and FNSC without utilizing any filters; 2) no high frequency chattering; 3 ) satisfactory DC component rejection;
4) comparable performance with DSOGI based sequence separation using measured voltage; 5) predictive ability to compensate one-step delay in predictive control.

FCS-MPPC is implemented based on SMGVO and tested on a two-level PWM rectifier to verify the effectiveness of the control system. Experimental results show that FPSC and FNSC can be accurately estimated and separated. The dynamic performance of SMGVO during voltage sag is similar to that of DSOGI. The implemented voltage sensorless FCSMPPC presents fast dynamic responses which can track power reference quickly. Direct start without initial knowledge of grid voltage is possible due to fast converging rate of SMGVO and high regulation bandwidth of FCS-MPPC.

\section{REFERENCES}

[1] Z. Zhang, H. Xu, M. Xue, Z. Chen, T. Sun, R. Kennel, and C. M. Hackl, "Predictive control with novel virtual-flux estimation for backto-back power converters," IEEE Trans. Ind. Electron., vol. 62, no. 5, pp. 2823-2834, May 2015.

[2] A. M. Razali, M. A. Rahman, G. George, and N. A. Rahim, "Analysis and design of new switching lookup table for virtual flux direct power control of grid-connected three-phase pwm ac - dc converter," IEEE Trans. Ind. Appl., vol. 51, no. 2, pp. 1189-1200, March 2015.

[3] J. Kukkola and M. Hinkkanen, "State observer for grid-voltage sensorless control of a converter equipped with an lcl filter: Direct discretetime design," IEEE Trans. Ind. Appl., vol. 52, no. 4, pp. 3133-3145, July 2016.

[4] H.-S. Song, I.-W. Joo, and K. Nam, "Source voltage sensorless estimation scheme for pwm rectifiers under unbalanced conditions," IEEE Trans. Ind. Electron., vol. 50, no. 6, pp. 1238-1245, Dec 2003.

[5] K. H. Ahmed, A. M. Massoud, S. J. Finney, and B. W. Williams, "Sensorless current control of three-phase inverter-based distributed generation," IEEE Trans. Power Del., vol. 24, no. 2, pp. 919-929, April 2009.

[6] M. B. Ketzer and C. B. Jacobina, "Multivariable load current sensorless controller for universal active power filter,' IET Power Electron., vol. 7 no. 7, pp. 1777-1786, July 2014.

[7] H. Kömürcügil and O. Kükrer, "A novel current-control method for three-phase pwm ac/dc voltage-source converters," IEEE Trans. Ind. Electron., vol. 46, no. 3, pp. 544-553, Jun 1999.

[8] M. B. Ketzer and C. B. Jacobina, "Virtual flux sensorless control for shunt active power filters with quasi-resonant compensators," IEEE Trans. Power Electron., vol. 31, no. 7, pp. 4818-4830, July 2016.

[9] Y. Cho and K. B. Lee, "Virtual-flux-based predictive direct power control of three-phase pwm rectifiers with fast dynamic response," IEEE Trans. Power Electron., vol. 31, no. 4, pp. 3348-3359, April 2016.

[10] J. A. Suul, A. Luna, P. Rodríguez, and T. Undeland, "Voltage-sensor-less synchronization to unbalanced grids by frequency-adaptive virtual flux estimation," IEEE Trans. Ind. Electron., vol. 59, no. 7, pp. 2910-2923, July 2012.

[11] J. G. Norniella, J. M. Cano, G. A. Orcajo, C. H. Rojas, J. F. Pedrayes, M. F. Cabanas, and M. G. Melero, "Improving the dynamics of virtualflux-based control of three-phase active rectifiers," IEEE Trans. Ind. Electron., vol. 61, no. 1, pp. 177-187, Jan 2014.

[12] S. Golestan, J. M. Guerrero, and G. B. Gharehpetian, "Five approaches to deal with problem of dc offset in phase-locked loop algorithms: Design considerations and performance evaluations," IEEE Trans. Power Electron., vol. 31, no. 1, pp. 648-661, Jan 2016.

[13] J. M. Kanieski, R. Cardoso, H. Pinheiro, and H. A. Grndling, "Kalman filter-based control system for power quality conditioning devices," IEEE Trans. Ind. Electron., vol. 60, no. 11, pp. 5214-5227, Nov 2013.

[14] R. Guzman, L. G. de Vicuña, J. Morales, M. Castilla, and J. Miret, "Model-based control for a three-phase shunt active power filter," IEEE Trans. Ind. Electron., vol. 63, no. 7, pp. 3998-4007, July 2016.

[15] H. Fehr and A. Gensior, "On trajectory planning, backstepping controller design and sliding modes in active front-ends," IEEE Trans. Power Electron., vol. 31, no. 8, pp. 6044-6056, Aug 2016.

[16] J. F. Silva, "Sliding-mode control of boost-type unity-power-factor pwm rectifiers," IEEE Trans. Ind. Electron., vol. 46, no. 3, pp. 594-603, Jun 1999. 
[17] R. G. Tonin, T. Bernardes, J. R. Massing, and H. Pinheiro, "Sliding mode observer for voltage sensorless current control of grid-connected converters," in 2013 Brazilian Power Electronics Conference, Oct 2013, pp. 387-392.

[18] J. D. Barros, J. F. A. Silva, and . G. A. Jesus, "Fast-predictive optimal control of npc multilevel converters," IEEE Trans. Ind. Electron., vol. 60, no. 2, pp. 619-627, Feb 2013.

[19] J. Scoltock, T. Geyer, and U. K. Madawala, "Model predictive direct power control for grid-connected npc converters," IEEE Trans. Ind. Electron., vol. 62, no. 9, pp. 5319-5328, Sept 2015.

[20] S. Kwak and S. k. Mun, "Model predictive control methods to reduce common-mode voltage for three-phase voltage source inverters," IEEE Trans. Power Electron., vol. 30, no. 9, pp. 5019-5035, Sept 2015.

[21] D. K. Choi and K. B. Lee, "Dynamic performance improvement of ac/dc converter using model predictive direct power control with finite control set," IEEE Trans. Ind. Electron., vol. 62, no. 2, pp. 757-767, Feb 2015.

[22] Y. Zhang and C. Qu, "Model predictive direct power control of pwm rectifiers under unbalanced network conditions," IEEE Trans. Ind. Electron., vol. 62, no. 7, pp. 4011-4022, July 2015.

[23] B. S. Riar, T. Geyer, and U. K. Madawala, "Model predictive direct current control of modular multilevel converters: Modeling, analysis, and experimental evaluation," IEEE Trans. Power Electron., vol. 30, no. 1, pp. 431-439, Jan 2015.

[24] Y. Zhang, J. Gao, and C. Qu, "Relationship between two direct power control methods for pwm rectifiers under unbalanced network," IEEE Trans. Power Electron., vol. 32, no. 5, pp. 4084-4094, May 2017.

[25] J. Rodriguez and P. Cortes, Predictive control of power converters and electrical drives. Wiley-IEEE Press, 2012, vol. 37.

[26] H. A. Young, M. A. Perez, J. Rodriguez, and H. Abu-Rub, "Assessing finite-control-set model predictive control: A comparison with a linear current controller in two-level voltage source inverters," IEEE Ind. Electron. Mag., vol. 8, no. 1, pp. 44-52, March 2014.

[27] M. Preindl and E. Schaltz, "Sensorless model predictive direct current control using novel second-order pll observer for pmsm drive systems," IEEE Trans. Ind. Electron., vol. 58, no. 9, pp. 4087-4095, Sept 2011.

[28] W. Gao and J. C. Hung, "Variable structure control of nonlinear systems: a new approach," IEEE Trans. Ind. Electron., vol. 40, no. 1, pp. 45-55, Feb 1993.

[29] W. Li, X. Ruan, C. Bao, D. Pan, and X. Wang, "Grid synchronization systems of three-phase grid-connected power converters: A complexvector-filter perspective," IEEE Trans. Ind. Electron., vol. 61, no. 4, pp. 1855-1870, April 2014

[30] Y. F. Wang and Y. W. Li, "Three-phase cascaded delayed signal cancellation pll for fast selective harmonic detection," IEEE Trans. Ind. Electron., vol. 60, no. 4, pp. 1452-1463, April 2013.

[31] S. Golestan, M. Monfared, F. D. Freijedo, and J. M. Guerrero, "Dynamics assessment of advanced single-phase pll structures," IEEE Trans. Ind. Electron., vol. 60, no. 6, pp. 2167-2177, June 2013

[32] Y. F. Wang and Y. W. Li, "Grid synchronization pll based on cascaded delayed signal cancellation," IEEE Trans. Power Electron., vol. 26, no. 7, pp. 1987-1997, July 2011.

[33] H. Yang, Y. Zhang, P. D. Walker, N. Zhang, and B. Xia, "A method to start rotating induction motor based on speed sensorless modelpredictive control," IEEE Trans. Energy Convers., vol. 32, no. 1, pp. 359-368, March 2017.

[34] P. Rodríguez, A. Luna, R. S. M. noz Aguilar, I. Etxeberria-Otadui, R. Teodorescu, and F. Blaabjerg, "A stationary reference frame grid synchronization system for three-phase grid-connected power converters under adverse grid conditions," IEEE Trans. Power Electron., vol. 27 , no. 1, pp. 99-112, Jan 2012.

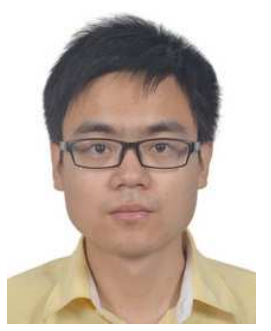

Haitao Yang (S'16) received the B.S. degree from Hefei University of Technology, Hefei, China, in 2009, and the M.S. degree from North China University of Technology, Beijing, China, in 2015, both in electrical engineering. He is currently working toward the Ph.D. degree in mechanical engineering with the School of Mechanical and Mechatronic Engineering, University of Technology, Sydney, NSW 2007, Australia.

His research interests include motor drives, posiconverters and electric vehicle.

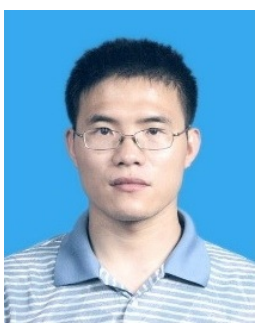

Yongchang Zhang (M'10) received the B.S. degree from Chongqing University, China, in 2004 and the $\mathrm{Ph} . \mathrm{D}$. degree from Tsinghua University, China, in 2009 , both in electrical engineering.

From August 2009 to August 2011, he was a Postdoctoral Fellow at the University of Technology Sydney, Australia. He joined North China University of Technology in August 2011 as an associate professor. Currently he is a full professor and the vice director of Inverter Technologies Engineering Research Center of Beijing. He has published more than 100 technical papers in the area of motor drives, pulsewidth modulation and $\mathrm{AC} / \mathrm{DC}$ converters. His current research interest is model predictive control for power converters and motor drives.

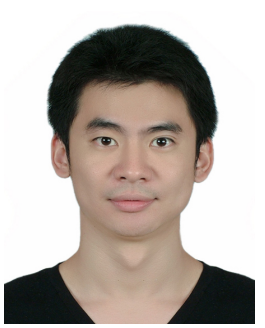

Jiejunyi Liang received the B.S. degree from Huazhong University of Science \& Technology, WuHan, China, in 2012 in Mechanical Design, Manufacturing and Automation, and the M.S. degree from University of Macau, Macau, China, in 2015 in Electromechanical Engineering. He is currently working toward the Ph.D. degree in Mechanical Engineering with the School of Mechanical and Mechatronic Engineering, University of Technology Sydney, NSW 2007, Australia.

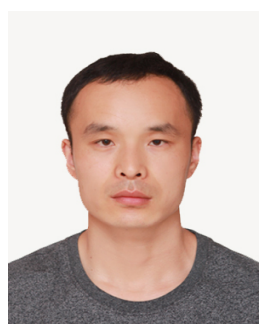

Jihao Gao was born in 1988. He received the B.S.degree from Henan University of Engineering, Zhengzhou, China, in 2013, and the M.S.degree from North China University of Technology, Beijing, China, in 2017, both in electrical engineering. $\mathrm{He}$ is currently an assistant engineer in the Power supply department of Bejing Great Well Eletronic Equipment Co.,Ltd.

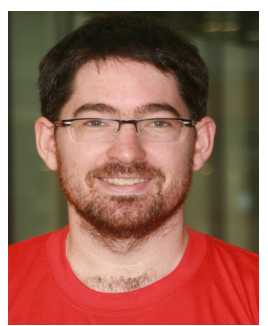

Paul D. Walker was born in Sydney, Australia, in 1981. He received the B.E. degree and the Ph.D. degree in mechanical engineering from the University of Technology Sydney (UTS), Sydney, Australia, in 2007 and 2011 respectively.

Since 2011, he has been a Research Associate and is currently a Senior Lecturer in the School of Mechanical and Mechatronic Engineering at UTS. His research interests include the development of novel power splitting transmissions for hybrid electric vehicles, multispeed transmission dynamics and control, and novel hybrid and electric vehicle topologies and their control.

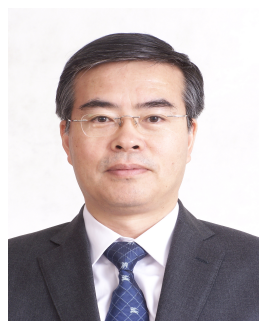

Nong Zhang received $\mathrm{PhD}$ in 1989 from the University of Tokyo and worked at several universities in China, Japan, USA and Australia before joining University of Technology, Sydney in 1995. Since 2009, he has been the Professor of Mechanical Engineering, at School of Mechanical and Mechatronic Engineering, University of Technology Sydney. He focused on fundamental research on mechanical vibration, multi-body system dynamics and its applications to complex machines and vehicular systems. He developed advanced models and numerical schemes for simulating gear shift in powertrains with AT, MT and CVTs and for dynamic analysis of vehicles fitted with advanced suspensions. 